\title{
Sliding Sector-Based Variable Structure Control of Continuous-Time Markov Jump Linear Systems Subject to Unknown Transition Rates
}

\author{
Yan-Mei Xue, ${ }^{1}$ Jianwei Yang, ${ }^{1}$ and Xiao-Mei Liu' ${ }^{2}$ \\ ${ }^{1}$ The School of Mathematics \& Statistics, Nanjing University of Information Science \& Technology, Nanjing, Jiangsu 210044, China \\ ${ }^{2}$ The School of Automation, Southeast University, Nanjing, Jiangsu 210096, China \\ Correspondence should be addressed to Yan-Mei Xue; ymxue1@163.com
}

Received 8 August 2013; Accepted 5 October 2013

Academic Editor: Tao Li

Copyright (C) 2013 Yan-Mei Xue et al. This is an open access article distributed under the Creative Commons Attribution License, which permits unrestricted use, distribution, and reproduction in any medium, provided the original work is properly cited.

\begin{abstract}
Based on sliding sector technique, the variable structure control for a class of uncertain continuous-time Markovian jump linear systems (MJLS) is investigated. The elements in the transition rate matrix include completely known, boundary known, and completely unknown ones. First, the related notions about sliding sector for continuous-time Markov jump linear systems are given; then based on linear matrix inequalities (LMIs) technique, sufficient conditions for the design of the sliding sector are provided. Second, a variable structure control law is presented to guarantee the mean-square quadratic stability of the closed-loop system in spite of the effects of the existing uncertainties and unknown/uncertain transition rates. Finally, an example is given to verify the validity of the theoretical results.
\end{abstract}

\section{Introduction}

The subject of this paper is variable structure control of continuous-time Markov jump linear uncertain systems. Control design problems of Markov jump systems are motivated by the fact that these systems represent a class of important stochastic systems which is popular in modeling practical engineering systems subject to failures or changes in structures and parameters. Such as in $[1,2]$, Markovian switching technique has been used to model the switching action of mobile manipulators. In addition, some important theoretical results have also been published so far; see, for example, [3-9] and the references therein. It is worth noticing that these results require that the transition probabilities/rates must be known precisely. In fact, the likelihood of obtaining the perfect information on all transition probabilities/rates is questionable, and the cost might be expensive in some areas. As a result, the study of control synthesis for Markov jump systems with uncertain or unknown transition probabilities/rates becomes interesting, and some important results have been established. For instance, the robust control designs for MJLS subject to the norm-bounded or polytopic uncertainties in the transition rates were considered in [10, 11]. However, they both require to know the structure and "nominal" terms of the uncertain transition rates. Furthermore, control designs of MJLS with partly unknown transition rates were developed in [12-14], in which the transition rate matrix included two kinds of elements: completely known and completely unknown ones. In fact, besides the two kinds of elements, it might also own boundary known elements in practice, and we will consider this more general case for reducing the conservatism of control design in this paper.

On the other hand, variable structure control is a wellknown robust control technique against external disturbances, parameter uncertainties, and unmodeled dynamics [15-17], and thus there are also some remarkable results published on variable structure control of continuous-time MJLS recently [18-22]. In [21], the robust stabilization of variable structure control for continuous-time MJLS with matched uncertainties was first investigated, and then in [22], a singular system approach was introduced for systems with parameter uncertainties and unknown nonlinear function. It is worth mentioning that these works require the transition rates to be 
precisely known a prior, and to the best of our knowledge, no results have been reported to variable structure control of continuous-time MJLS with unknown transition rates. In addition, one of the disadvantages about variable structure control is that chattering phenomenon might occur on the neighbor of the sliding manifold which is harmful to actuator devices, and thus it is a barrier for the use of the variable structure control strategy [23-25]. In order to avoid the occurrence of chattering phenomenon, it is worth noting that for a class of linear deterministic systems, a variable structure controlbased sliding sector is first introduced in [26]. The main feature of the sliding sector-based variable structure control scheme is that zero control input can be used in the sliding sector of the state space and then the chattering phenomenon is avoided.

Motivated by the aforementioned discussion, for a class of continuous-time uncertain MJLS subject to unknown transition rates, a novel robust variable structure controlbased sliding sector technique is investigated. The main contribution of this paper is summarized as follows. First, related notions of the sliding sector for MJLS, $P_{i} R_{i}$-sliding sector, simplified $P_{i} R_{i}$-sliding sector, and inner sector and outer sector are given. Second, sufficient conditions for the design of the simplified $P_{i} R_{i}$-sliding sector for the considered Markov jump linear uncertain system are presented in terms of LMI technique. Finally, a variable structure control law is established to drive the state trajectory to converge to the inner sector of the $P_{i} R_{i}$-sliding sector and guarantee mean square quadratic stability of the closed-loop system in spite of the effect of unknown transition rates and system uncertainties.

The rest of this paper is organized as follows. The problem statement and preliminaries are presented in Section 2. The main results are given in Section 3. In Section 4, a numerical example is presented to illustrate the effectiveness of the results, and the conclusions are drawn in Section 5.

Throughout this paper, the following notations are used. $\mathbb{R}^{n}$ denotes the $n$-dimensional Euclidean space; $A^{T}$ stands for the transpose of matrix $A$; $I$ and 0 represent the identity matrix and zero matrix in appropriate dimension, respectively. In addition, the symbol $\mathrm{He}(X)$ is used to represent $X+$ $X^{T} . P>0(P \geq 0)$ means that $P$ is real symmetric and (semi-) positive definite. $|\cdot|$ denotes the absolute value of a scalar, the standard Euclidean norm of a vector, or the induced norm of a matrix, respectively. In symmetric block matrices, the notation $*$ is used to represent a term that is induced by symmetry.

\section{Problem Statement and Preliminaries}

Consider the following time-invariant continuous-time linear uncertain system:

$$
\dot{x}=\left(A\left(r_{t}\right)+\Delta A\left(r_{t}\right)\right) x+\left(B\left(r_{t}\right)+\Delta B\left(r_{t}\right)\right) u,
$$

where $x(t) \in \mathbb{R}^{n}$ and $u(t) \in \mathbb{R}$ are state and control input, respectively. $\Delta A r_{t}$ and $\Delta B\left(r_{t}\right)$ are the system uncertainties. $\left\{r_{t}, t \geq 0\right\}$ is a continuous-time Markovian process with right continuous trajectories and taking values in a finite set $\mathcal{S}=$ $\{1,2, \ldots, \mathbb{N}\}$. It governs the switching among the different system modes with the following mode transition probabilities:

$$
\operatorname{Prob}\left\{r_{t+m}=j \mid r_{t}=i\right\}= \begin{cases}\lambda_{i j} m+o(m), & \text { if } j \neq i, \\ 1+\lambda_{i i} m+o(m), & \text { if } j=i,\end{cases}
$$

where $m>0, \lim _{m \rightarrow 0}(o(m) / m)=0$ and $\lambda_{i j} \geq 0(i, j \in$ $\delta, j \neq i$ ) denote the switching rate from mode $i$ at time $t$ to mode $j$ at time $t+m$, and $\lambda_{i i}=-\sum_{j=1, j \neq i}^{\mathbb{N}} \lambda_{i j}$ for each $i \in \mathcal{S}$.

In general, the Markov process transition rate matrix $\Lambda$ is defined by

$$
\Lambda=\left[\lambda_{i j}\right]_{\mathbb{N} \times \mathbb{N}} \cdot
$$

In this paper, the transition rates of the jumping process are assumed to be partly available; that is, some elements in $\Lambda$ have been exactly known; some ones are uncertain but with known boundaries; others may have no information to use. For instance, for system (1) with four operation modes, the transition rate matrix might be described by

$$
\Lambda=\left[\begin{array}{cccc}
? & \lambda_{12} & \lambda_{13} & ? \\
\lambda_{21} & ? & \lambda_{23} & ? \\
? & a & ? & \lambda_{34} \\
b & ? & \lambda_{43} & \lambda_{44}
\end{array}\right]
$$

where ? represents the completely unknown element in the transition rate matrix; parameters $a$ and $b$ represent the elements with known lower and upper bounds. That is, $\underline{a} \leq a \leq$ $\bar{a}$ and $\underline{b} \leq b \leq \bar{b}$, where $\underline{a}, \bar{a}, \underline{b}$, and $\bar{b}$ are known parameters; $\lambda_{i j}$ denotes the precisely known element.

For clarity, denote $\mathcal{S}=\mathcal{S}_{k 1}^{i} \cup \mathcal{S}_{k 2}^{i} \cup \mathcal{S}_{u k}^{i}, i=1,2, \ldots, \mathbb{N}$ by

$$
\begin{gathered}
\mathcal{S}_{k 1}^{i}=\left\{j: \lambda_{i j} \text { is exactly known }\right\}, \\
\mathcal{S}_{k 2}^{i}=\left\{j: \text { the bounds of } \lambda_{i j} \text { is known }\right\}, \\
\mathcal{S}_{u k}^{i}=\left\{j: \text { there is no information available for } \lambda_{i j}\right\} .
\end{gathered}
$$

Furthermore, let $\delta_{k}^{i}=\left\{j: \underline{\lambda}_{i j} \leq \lambda_{i j} \leq \bar{\lambda}_{i j}\right\}$; then $\mathcal{S}_{k}^{i}=\mathcal{S}_{k 1}^{i} \cup \mathcal{S}_{k 2}^{i}$. Let $\mathcal{S}_{k}^{i}=\left\{\mathscr{K}_{1}^{i}, \mathscr{K}_{2}^{i}, \ldots, \mathscr{K}_{m_{k}^{i}}^{i}\right\} . \quad \mathcal{S}_{u k}^{i}=$ $\left\{\mathbb{K}_{1}^{i}, \mathbb{K}_{2}^{i}, \ldots, \mathbb{K}_{m_{u k}^{i}}^{i}\right\}$, where $\mathscr{K}_{l_{1}}^{i}$ denotes the $l_{1}$ th element in $\mathcal{S}_{k}^{i}$ with the index $\mathscr{K}_{l_{1}}^{i}$ in the $i$ th row of the matrix $\Lambda$. $\mathbb{K}_{l_{2}}^{i}$ denotes the $l_{2}$ th element in $\delta_{u k}^{i}$ with the index $\mathbb{K}_{l_{2}}^{i}$ in the $i$ th row of the matrix $\Lambda . m_{k}^{i}$ and $m_{u k}^{i}$ represent the number of elements in $\mathcal{S}_{k}^{i}$ and $\delta_{u k}^{i}$, respectively.

Remark 1 . When $\underline{\lambda}_{i j}=\bar{\lambda}_{i j}$, the transition rates are reduced to the situation in [12]. Obviously, the solving method there can only treat the above known bound case as the completely unknown case, and some conservativeness has been brought in.

For convenience, let $A_{i}=A\left(r_{t}\right), \Delta A_{i}=\Delta A\left(r_{t}\right), B_{i}=$ $B\left(r_{t}\right)$, and $\Delta B_{i}=\Delta B\left(r_{t}\right)$ for each possible value $r_{t}=i, i \in \mathcal{S}$, 
where $A_{i}$ and $B_{i}$ are known constant matrices with appropriate dimensions; then system (1) can be described by

$$
\dot{x}=\left(A_{i}+\Delta A_{i}\right) x+\left(B_{i}+\Delta B_{i}\right) u .
$$

Throughout this paper, for each $i$, suppose that the following assumptions are valid.

Assumption 2. The pair $\left(A_{i}, B_{i}\right)$ is controllable.

Assumption 3. The uncertainties satisfy $\Delta A_{i}=D_{i} E_{i}(t, x) F_{i}$, $\Delta B_{i}=B_{i} \Theta_{i}(t)$, where $D_{i}$ and $F_{i}$ are known constant matrices with appropriate dimensions, $E_{i}(t, x)$ is time-varying uncertain matrix satisfying $E_{i} E_{i}^{T} \leq I$, the parameter $\Theta_{i}(t)$ satisfies $\left|\Theta_{i}(t)\right| \leq \bar{d}_{i}<1, \bar{d}_{i}$ is a known positive scalar.

For continuous-time Markov jump linear nominal systems

$$
\dot{x}=A_{i} x+B_{i} u,
$$

some related notions of sliding sector are firstly presented.

Definition 4. The $P_{i} R_{i}$-sliding sector is a subset of $\mathbb{R}^{n}$ defined as

$$
\begin{gathered}
\mathcal{S}_{i}=\left\{x \mid x^{T}\left(A_{i}^{T} P_{i}+P_{i} A_{i}+\sum_{j=1}^{\mathbb{N}} \lambda_{i j} P_{j}\right) x\right. \\
\left.\leq-x^{T} R_{i} x, x \in \mathbb{R}^{n}\right\},
\end{gathered}
$$

where $P_{i} \in \mathbb{R}^{n \times n}$ is a positive definite symmetric matrix, $R_{i} \in$ $\mathbb{R}^{n \times n}$ is a positive semidefinite symmetric matrix, $R_{i}=C_{i}^{T} C_{i}$, $C_{i} \in \mathbb{R}^{l_{i} \times n}, l_{i} \geq 1$, and $\left(C_{i}, A_{i}\right)$ is an observable pair.

The set of the $P_{i} R_{i}$-sliding sector is nonempty since at least zero state stays inside it.

Obviously, while the state of the plant evolves in the $P_{i} R_{i^{-}}$ sliding sector $\mathcal{S}_{i}$, the $P_{i}$-norm $|x|_{P_{i}}=x^{T} P_{i} x$ of the plant (7) decreases with zero control input since

$$
\begin{aligned}
\dot{L}_{i} & =x^{T}\left(A_{i}^{T} P_{i}+P_{i} A_{i}+\sum_{j=1}^{\mathbb{N}} \lambda_{i j} P_{j}\right) x \\
& \leq-x^{T} R_{i} x \leq 0, \quad \forall x \in \mathcal{S}_{i}, \quad x \neq 0,
\end{aligned}
$$

where $L_{i}=|x|_{P_{i}}^{2}$.

Remark 5. When $A_{i}=A, P_{i}=P, R_{i}=R$, the $P_{i} R_{i}$-sliding sector in (8) becomes

$$
\mathcal{S}=\left\{x \mid x^{T}\left(A^{T} P+P A\right) x \leq-x^{T} R x, x \in \mathbb{R}^{n}\right\} .
$$

The definition of $P R$-sliding sector for linear deterministic systems is introduced in [26].
Definition 6. A simplified $P_{i} R_{i}$-sliding sector is a subset of $\mathbb{R}^{n}$ defined as

$$
\mathcal{S}_{i}=\left\{x|| s_{i}(x) \mid \leq \delta_{i}(x), x \in \mathbb{R}^{n}\right\},
$$

where the linear function $s_{i}(x)$ and the square root $\delta_{i}(x)$ of the quadratic function $\delta_{i}^{2}(x)$ are determined by

$$
\begin{gathered}
s_{i}(x)=S_{i} x, \quad S_{i} \in \mathbb{R}^{1 \times n}, \\
\delta_{i}(x)=\sqrt{x^{T} \Omega_{i} x}, \quad \Omega_{i} \in \mathbb{R}^{n \times n}, \Omega_{i} \geq 0\left(\Omega_{i} \neq 0\right) .
\end{gathered}
$$

Remark 7. Suppose that the following inequalities are satisfied:

$$
\begin{array}{r}
A_{i}^{T} P_{i}+P_{i} A_{i}+\sum_{j=1}^{\mathbb{N}} \lambda_{i j} P_{j}-P_{i} B_{i} B_{i}^{T} P_{i}+Q_{i}<0, \\
i=1,2, \ldots, \mathbb{N} .
\end{array}
$$

Then the parameters in the simplified $P_{i} R_{i}$-sliding sector $P_{i}$, $R_{i}, S_{i}$, and $\Omega_{i}$ can be selected as follows:

$$
\Omega_{i}=r_{i} Q_{i}, \quad R_{i}=\left(1-r_{i}\right) Q_{i}, \quad S_{i}=B_{i}^{T} P_{i},
$$

where $0<r_{i}<1$.

Obviously, while the state of the plant evolves in the simplified $P_{i} R_{i}$-sliding sector $\mathcal{S}_{i}$, the $P_{i}$-norm $|x|_{P_{i}}$ of the plant (7) decreases with zero control input since

$$
\begin{aligned}
\dot{L}_{i} & =x^{T}\left(A_{i}^{T} P_{i}+P_{i} A_{i}+\sum_{j=1}^{\mathbb{N}} \lambda_{i j} P_{j}\right) x \\
& =x^{T}\left(P_{i} B_{i} B_{i}^{T} P_{i}-Q_{i}\right) x \\
& =s_{i}^{2}(x)-\delta_{i}^{2}(x)-x^{T} R_{i} x \\
& \leq-x^{T} R_{i} x, \quad \forall x \in \mathcal{S}_{i}, \quad x \neq 0 .
\end{aligned}
$$

In addition, in order to avoid the chattering phenomenon on the boundary of the $P_{i} R_{i}$-sliding sector, an inner sector $\mathcal{S}_{i}^{\text {in }}$ and an outer sector $\mathcal{S}_{i}^{\text {out }}$ are introduced as subsets of the $P_{i} R_{i}{ }^{-}$ sliding sector (11):

$$
\begin{gathered}
\mathcal{S}_{i}^{\text {in }}=\left\{x|| s_{i}(x) \mid \leq \alpha_{i} \delta_{i}(x), x \in \mathbb{R}^{n}\right\}, \\
\mathcal{S}_{i}^{\text {out }}=\left\{x\left|\alpha_{i} \delta_{i}(x)<\right| s_{i}(x) \mid \leq \delta_{i}(x), x \in \mathbb{R}^{n}\right\},
\end{gathered}
$$

where the positive scalar $\alpha_{i}$ satisfies $0<\alpha_{i}<1$. It is not difficult to see that the sets $\mathcal{S}_{i}^{\text {in }}$ and $\mathcal{S}_{i}^{\text {out }}$ constitute a partition of the simplified $P R$-sliding sector $\mathcal{S}_{i}$. That is, $\mathcal{S}_{i}=\mathcal{S}_{i}^{\text {in }} \cup \mathcal{S}_{i}^{\text {out }}$ and $\mathcal{S}_{i}^{\text {in }} \cap \mathcal{S}_{i}^{\text {out }}=\emptyset$.

A hysteresis dead-zone function $\sigma_{i}\left(s_{i}(x), \delta_{i}(x)\right)$ is further introduced for avoiding the chattering. It is defined as

$$
\sigma_{i}\left(s_{i}(x), \delta_{i}(x)\right)= \begin{cases}0, & x \in \mathcal{S}_{i}^{\text {in }} \\ \text { unchanged, }, & x \in \mathcal{S}_{i}^{\text {out }} \\ 1, & x \in \mathcal{S}_{i} .\end{cases}
$$


Lemma 8 (see [20]). For any given $\lambda_{j} \geq 0$ and matrices $P_{j}>$ $0\left(P_{j} \in R^{n \times n}, 1 \leq j \leq \mathbb{N}\right)$, if there exists $Z_{i} \geq P_{j}$, then the following inequality holds:

$$
\sum_{j=1}^{\mathbb{N}} \lambda_{j} P_{j} \leq \sum_{j=1}^{\mathbb{N}} \lambda_{j} Z_{i}
$$

Lemma 9 (see [27]). Given a symmetric matrix $\Pi$ and matrices $M, N$ with appropriate dimensions, then $\Pi+M F(t) N+$ $N^{T} F^{T}(t) M^{T}<0$ for all $F(t)$ satisfying $F^{T}(t) F(t) \leq I$, if and only if there exists a scalar $\varepsilon>0$ such that the following inequality holds:

$$
\Pi+\varepsilon M M^{T}+\varepsilon^{-1} N^{T} N<0 .
$$

Lemma 10 (see [28]). For symmetric and positive definite matrices $X$ and $Y$, if $X \geq Y>0$, then $Y^{-1} \geq X^{-1}>0$.

\section{Main Results}

First, we present the design of the simplified $P_{i} R_{i}$-sliding sector for the considered continuous-time Markov jump linear uncertain system (6). Second, the controller design for meansquare quadratic stability of the continuous-time Markov jump linear uncertain system is developed.

\subsection{The Design of the Simplified $P_{i} R_{i}$-Sliding Sector}

Theorem 11. For continuous-time Markov jump linear uncertain system (6), there exist symmetric and positive definite matrices $X_{i}, Z_{i}$, and $Y_{i}$ satisfying the following LMIs:

$$
\begin{gathered}
i \in \mathcal{S}_{k}^{i},\left\{\begin{array}{c}
{\left[\begin{array}{ccccc}
\Gamma_{11 i} & \Gamma_{12 i} & \Gamma_{13 i} & X_{i} & X_{i} F_{i} \\
* & -\Gamma_{22 i} & 0 & 0 & 0 \\
* & * & -Z_{i} & 0 & 0 \\
* & * & * & -Y_{i} & 0 \\
* & * & * & * & -\varepsilon_{i} I
\end{array}\right]<0,} \\
Z_{i} \leq X_{j}, j \in \mathcal{S}_{u k}^{i}, \quad j \neq i
\end{array}\right. \\
i \in \mathcal{S}_{u k}^{i},\left\{\begin{array}{cccc}
\Phi_{11 i} & \Phi_{12 i} & X_{i} & X_{i} F_{i} \\
* & -X_{j} & 0 & 0 \\
* & * & -Y_{i} & 0 \\
* & * & * & -\varepsilon_{i} I
\end{array}\right]<0, \\
X_{i} \leq X_{j}, j \in \mathcal{S}_{u k}^{i}, j \neq i,
\end{gathered}
$$

where

$$
\begin{aligned}
\Gamma_{11 i} & =\operatorname{He}\left(A_{i} X_{i}\right)+\bar{\lambda}_{i i} X_{i}-B_{i} B_{i}^{T}+\varepsilon_{i} D_{i} D_{i}^{T}, \\
\Gamma_{12 i} & =\left[\sqrt{\bar{\lambda}_{i \mathscr{K}_{1}^{i}}} X_{i}, \sqrt{\bar{\lambda}_{i \mathscr{K}_{2}^{i}}} X_{i}, \ldots, \sqrt{\bar{\lambda}_{i \mathscr{K}_{m_{k}^{i}}^{i}}} X_{i}\right], \\
\Gamma_{22 i} & =\operatorname{diag}\left\{-X_{\mathscr{K}_{1}^{i}},-X_{\mathscr{K}_{2}^{i}}, \ldots,-X_{\mathscr{K}_{m_{k}^{i}}^{i}}\right\}, \\
\Gamma_{13 i} & =\sqrt{-\left(\sum_{j \in \mathcal{S}_{k}^{i}, j \neq i} \underline{\lambda}_{i j}+\underline{\lambda}_{i i}\right)} X_{i}, \\
\Phi_{11 i} & =\operatorname{He}\left(A_{i} X_{i}\right)-\sum_{j \in \delta_{k}^{i}, j \neq i} \underline{\lambda}_{i j} X_{i}-B_{i} B_{i}^{T}+\varepsilon_{i} D_{i} D_{i}^{T},
\end{aligned}
$$

$$
\begin{aligned}
& \Phi_{12 i}=\left[\sqrt{\bar{\lambda}_{i \mathscr{K}_{1}^{i}}} X_{i}, \sqrt{\bar{\lambda}_{i \mathscr{K}_{2}^{i}}} X_{i}, \ldots, \sqrt{\bar{\lambda}_{\mathscr{C}_{\mathscr{K}^{i}}^{i}}} X_{i}\right], \\
& \Phi_{22 i}=\operatorname{diag}\left\{-X_{\mathscr{K}_{1}^{i}},-X_{\mathscr{K}_{2}^{i}}, \ldots,-X_{\mathscr{K}_{m_{k}^{i}}^{i}}\right\} ;
\end{aligned}
$$

then the parameters of $P_{i} R_{i}$-sliding sector in (14) can be designed as $P_{i}=X_{i}^{-1}, Q_{i}=Y_{i}^{-1}, \Omega_{i}=r_{i} Y_{i}^{-1}, R_{i}=\left(1-r_{i}\right) Y_{i}^{-1}$, and $S_{i}=B_{i}^{T} X_{i}^{-1}$.

Proof. For system (6), for all $\Delta A_{i}, i=1,2, \ldots, \mathbb{N}$, the conditions in (13) are equal to

$$
\begin{gathered}
\left(A_{i}+\Delta A_{i}\right)^{T} P_{i}+P_{i}\left(A_{i}+\Delta A_{i}\right) \\
+\sum_{j \in \delta_{k}^{i}, j \neq i} \lambda_{i j} P_{j}+\sum_{j \in \delta_{k k, j}^{i}, j \neq i} \lambda_{i j} P_{j} \\
+\lambda_{i i} P_{i}-P_{i} B_{i} B_{i}^{T} P_{i}+Q_{i}<0 .
\end{gathered}
$$

In the following, we consider two cases: $i \in \mathcal{S}_{k}^{i}$ and $i \in \mathcal{S}_{u k}^{i}$ in the proof.

Case I $\left(i \in \delta_{k}^{i}\right)$. In this case, the conditions in (24) can be guaranteed when for $i=1,2, \ldots, \mathbb{N}$, the following inequalities:

$$
\begin{gathered}
\text { He }\left(\left(A_{i}+\Delta A_{i}\right)^{T} P_{i}\right)+\sum_{j \in \mathcal{S}_{k}^{i}, j \neq i} \lambda_{i j} P_{j} \\
+\sum_{j \in \delta_{u k}^{i}, j \neq i} \lambda_{i j} W_{i}+\lambda_{i i} P_{i} \\
-P_{i} B_{i} B_{i}^{T} P_{i}+Q_{i}<0, \\
\quad P_{j} \leq W_{i}, \quad j \in \mathcal{S}_{u k}^{i},
\end{gathered}
$$

are satisfied. Substituting $\sum_{j \in \mathcal{S}_{u k}^{i}, j \neq i} \lambda_{i j}=-\sum_{j \in \mathcal{S}_{k}^{i}, j \neq i} \lambda_{i j}-\lambda_{i i}$ into the first inequality above and utilizing the boundary information of $\lambda_{i j}$, one can see that the above inequalities can be guaranteed by

$$
\begin{gathered}
\operatorname{He}\left(\left(A_{i}+\Delta A_{i}\right)^{T} P_{i}\right) \\
+\sum_{j \in \mathcal{S}_{k}^{i}, j \neq i} \bar{\lambda}_{i j} P_{j}-\left(\sum_{j \in \mathcal{S}_{k}^{i}, j \neq i} \underline{\lambda}_{i j}+\underline{\lambda}_{i i}\right) W_{i} \\
+\bar{\lambda}_{i i} P_{i}-P_{i} B_{i} B_{i}^{T} P_{i}+Q_{i}<0, \\
P_{j} \leq W_{i}, \quad j \in \mathcal{S}_{u k}^{i} .
\end{gathered}
$$

Pre- and postmultiplying $P_{i}^{-1}$ on both sides of the first inequality in above inequalities and letting $X_{i}=P_{i}^{-1}$, one has

$$
\begin{aligned}
\operatorname{He} & \left(\left(A_{i}+\Delta A_{i}\right) X_{i}\right)+\sum_{j \in \delta_{k}^{i}, j \neq i} \bar{\lambda}_{i j} X_{i} X_{j}^{-1} X_{i} \\
& -\left(\sum_{j \in \delta_{k}^{i}, j \neq i} \underline{\lambda}_{i j}+\underline{\lambda}_{i i}\right) X_{i} W_{i} X_{i}+\bar{\lambda}_{i i} X_{i} \\
& -B_{i} B_{i}^{T}+X_{i} Q_{i} X_{i}<0 .
\end{aligned}
$$


Let $Y_{i}=Q_{i}^{-1}, Z_{i}=W_{i}^{-1}$, and applying Schur-complement technique and Lemma 9, one can obtain the first LMI condition in (21).

In addition, by using Lemma 10, one can see that the second inequality in (26) can be converted into the second LMI condition in (21).

Case II $\left(i \in \delta_{u k}^{i}\right)$. In this case, substituting $\lambda_{i i}=$ $-\sum_{j \in \mathcal{S}_{k}^{i}, j \neq i} \lambda_{i j}-\sum_{j \in \mathcal{S}_{u k}^{i}, j \neq i} \lambda_{i j}$ into (24), one has

$$
\begin{aligned}
& \operatorname{He}\left(\left(A_{i}+\Delta A_{i}\right)^{T} P_{i}\right)+\sum_{j \in \mathcal{S}_{k}^{i}, j \neq i} \lambda_{i j} P_{j} \\
& +\sum_{j \in \delta_{u k}^{i}, j \neq i} \lambda_{i j} P_{j}-\left(\sum_{j \in \mathcal{S}_{k}^{i}, j \neq i} \lambda_{i j}+\sum_{j \in \delta_{u k}^{i}, j \neq i} \lambda_{i j}\right) P_{i} \\
& -P_{i} B_{i} B_{i}^{T} P_{i}+Q_{i}<0, \\
& \quad i=1,2, \ldots, \mathbb{N} .
\end{aligned}
$$

The conditions in (28) can be guaranteed provided that for $i=1,2, \ldots, \mathbb{N}$, one has

$$
\begin{gathered}
\mathrm{He}\left(\left(A_{i}+\Delta A_{i}\right)^{T} P_{i}\right)+\sum_{j \in \mathcal{S}_{k}^{i}, j \neq i} \lambda_{i j} P_{j} \\
-\sum_{j \in \mathcal{S}_{k}^{i}, j \neq i} \lambda_{i j} P_{i}-P_{i} B_{i} B_{i}^{T} P_{i}+Q_{i}<0, \\
P_{j} \leq P_{i}, \quad j \in \mathcal{S}_{u k}^{i}, j \neq i .
\end{gathered}
$$

Taking use of the boundary information of $\lambda_{i j}$, one can see that the above inequalities can be guaranteed by

$$
\begin{gathered}
\mathrm{He}\left(\left(A_{i}+\Delta A_{i}\right)^{T} P_{i}\right)+\sum_{j \in \mathcal{S}_{k}^{i}, j \neq i} \bar{\lambda}_{i j} P_{j} \\
-\sum_{j \in \mathcal{S}_{k}^{i}, j \neq i} \lambda_{i j} P_{i}-P_{i} B_{i} B_{i}^{T} P_{i}+Q_{i}<0, \\
P_{j} \leq P_{i}, \quad j \in \mathcal{S}_{u k}^{i}, \quad j \neq i .
\end{gathered}
$$

Pre- and postmultiplying $P_{i}^{-1}$ on both sides of the first inequality in above inequalities and letting $X_{i}=P_{i}^{-1}$ and $Y_{i}=$ $Q_{i}^{-1}$, one has

$$
\begin{array}{r}
\mathrm{He}\left(\left(A_{i}+\Delta A_{i}\right) X_{i}\right)+\sum_{j \in \mathcal{S}_{k}^{i}, j \neq i} \bar{\lambda}_{i j} X_{i} X_{j}^{-1} X_{i} \\
-\sum_{j \in \mathcal{S}_{k}^{i}, j \neq i} \underline{\lambda}_{i j} X_{i}-B_{i} B_{i}^{T}+X_{i} \bar{Q}_{i}^{-1} X_{i}<0 .
\end{array}
$$

Applying Schur-complement technique and Lemma 9 again, the above inequality (31) can be converted into the first LMI condition in (22).

Applying Lemma 10, one can see that the second inequality in (30) can be converted into the second condition in (22). By the design of the parameters of PR-sliding sector in (14), the proof of Theorem 11 is completed.

\subsection{Variable Structure Control Design}

Theorem 12. Considering the simplified $P_{i} R_{i}$-sliding sector $\mathcal{S}_{i}$ (11) with the inner sector $\mathcal{S}_{i}^{\text {in }}(16)$ and the outer sector $\mathcal{S}_{i}^{\text {out }}(17)$, the following variable structure controller is designed to ensure that the state trajectories of the plant (6) enter into the inner sector from the outside of $P_{i} R_{i}$-sliding sector and obtain meansquare quadratic stability of the closed-loop system. Consider

$$
u=u_{i 1}+u_{i 2},
$$

where

$$
\begin{aligned}
u_{i 1}=-\sigma_{i}\left(s_{i}(x),\right. & \left.\delta_{i}(x)\right)\left(S_{i} B_{i}\right)^{-1}\left(S_{i} A_{i} x+K_{i} s_{i}(x)\right), \\
u_{i 2}= & -\frac{1}{1-\bar{d}_{i}} \sigma_{i}\left(s_{i}(x), \delta_{i}(x)\right) \\
& \times\left(S_{i} B_{i}\right)^{-1} \operatorname{sign}\left(s_{i}(x)\right) \rho_{i},
\end{aligned}
$$

$\rho_{i}=\left|S_{i}\right|\left|D_{i}\right|\left|F_{i}\right||x|+\bar{d}_{i}\left|S_{i} A_{i}\right||x|+\bar{d}_{i} K_{i}\left|s_{i}(x)\right|$ and the parameter $K_{i}>\max \left\{S_{i} B_{i} / 2, H_{i}\right\}$ satisfies the following inequality:

$$
2 H_{i} \alpha_{i}^{2} r_{i} Q_{i}+S_{i}^{T} S_{i} A_{i}+A_{i}^{T} S_{i}^{T} S_{i}>0 .
$$

Proof. It is assumed that the initial state of the plant lies outside the $P_{i} R_{i}$-sliding sector. In this case, we have $\sigma_{i}\left(s_{i}(x), \delta_{i}(x)\right)=1$ and $u_{i 1}=-\left(S_{i} B_{i}\right)^{-1}\left(S_{i} A_{i} x+K_{i} s_{i}(x)\right)$. It can be easily checked that

$$
\begin{aligned}
s_{i}(x) \dot{s}_{i}(x)= & -K_{i} s_{i}^{2}(x)+s_{i}(x) S_{i} \Delta A_{i} x \\
& +s_{i}(x) S_{i} B_{i} u_{i 2}+s_{i}(x) S_{i} \Delta B_{i} u .
\end{aligned}
$$

Noting that $\Delta B_{i}=B_{i} \Theta_{i}$ and $|\Theta| \leq \bar{d}_{i}<1$, we have

$$
\begin{aligned}
s_{i}(x) \dot{s}_{i}(x) \leq & -K_{i} s_{i}^{2}(x)+s_{i}(x) S_{i} B_{i} u_{i 2} \\
& +\left|s_{i}(x)\right|\left|S_{i}\right|\left|\Delta A_{i}\right||x|+\bar{d}_{i}\left|s_{i}(x)\right|\left|S_{i} B_{i}\right||u| .
\end{aligned}
$$

In view of $u=u_{i 1}+u_{i 2}$, it is easy to check that

$$
\begin{aligned}
s_{i}(x) \dot{s}_{i}(x) \leq & -K_{i} s_{i}^{2}(x)+s_{i}(x) S_{i} B_{i} u_{i 2} \\
& +\left|s_{i}(x)\right|\left|S_{i}\right|\left|\Delta A_{i}\right||x|+\bar{d}_{i}\left|s_{i}(x)\right| \\
& \times\left|S_{i} B_{i}\right|\left|u_{i 1}\right|+\bar{d}_{i}\left|s_{i}(x)\right|\left|S_{i} B_{i}\right|\left|u_{i 2}\right| .
\end{aligned}
$$

Substituting $u_{i 1}=-\left(S_{i} B_{i}\right)^{-1}\left(S_{i} A_{i} x+K_{i} s_{i}(x)\right)$ into the above inequality and noting that $S_{i} B_{i}=B_{i}^{T} P_{i} B_{i}>0$, one can see that

$$
\begin{aligned}
s_{i}(x) & \dot{s}_{i}(x) \\
\leq & -\left(1-\bar{d}_{i}\right) K_{i} s_{i}^{2}(x)+\left(1-\bar{d}_{i}\right) s_{i}(x) S_{i} B_{i} u_{i 2} \\
& +\left|s_{i}(x)\right|\left|S_{i}\right|\left|\Delta A_{i}\right||x|+\bar{d}_{i}\left|s_{i}(x)\right|\left|S_{i} A_{i}\right| \\
& \times|x|+\bar{d}_{i} s_{i}(x) S_{i} B_{i} u_{i 2}+\bar{d}_{i}\left|s_{i}(x)\right|\left|S_{i} B_{i}\right|\left|u_{i 2}\right| .
\end{aligned}
$$


Taking use of $u_{i 2}$ in (34) where $\rho_{i}=\left|S_{i}\right|\left|D_{i}\right|\left|F_{i}\right||x|+$ $\bar{d}_{i}\left|S_{i} A_{i}\right||x|+\bar{d}_{i} K_{i}\left|s_{i}(x)\right|>\bar{d}_{i}\left|S_{i} A_{i}\right||x|+\bar{d}_{i} K_{i}\left|s_{i}(x)\right|$, one can see that

$$
\begin{aligned}
s_{i}(x) \dot{s}_{i}(x) \leq & -K_{i} s_{i}^{2}(x)+\bar{d}_{i} s_{i}(x) S_{i} B_{i} u_{i 2} \\
& +\bar{d}_{i}\left|s_{i}(x)\right|\left|S_{i} B_{i}\right|\left|u_{i 2}\right| .
\end{aligned}
$$

According to the design of $u_{i 2}$ again, one can easily observe that

$$
\begin{aligned}
& s_{i}(x) S_{i} B_{i} u_{i 2}+\left|s_{i}(x)\right|\left|S_{i} B_{i}\right|\left|u_{i 2}\right| \\
& \leq-\frac{1}{1-\bar{d}_{i}}\left|s_{i}(x)\right| \rho_{i}+\frac{1}{1-\bar{d}_{i}}\left|s_{i}(x)\right| \\
& \quad \times\left|S_{i} B_{i}\right|\left|\left(S_{i} B_{i}\right)^{-1}\right|\left|\operatorname{sign}\left(s_{i}(x)\right)\right| \rho_{i}=0 .
\end{aligned}
$$

It follows from (40) and (41) that

$$
s_{i}(x) \dot{s}_{i}(x) \leq-K_{i} s_{i}^{2}(x) .
$$

Thus the state trajectory of the closed-loop system will be driven into the inner sector of the $P_{i} R_{i}$-sliding sector.

In the following, we will prove that the $P_{i}$-norm keeps decreasing. Consider

$$
\begin{aligned}
\dot{L}_{i}(t)= & x^{T}\left\{\left(A_{i}+\Delta A_{i}\right)^{T} P+P\left(A_{i}+\Delta A_{i}\right)+\sum_{j=1}^{\mathbb{N}} \lambda_{i j} P_{j}\right\} x \\
& +2 x^{T} P_{i}\left(B_{i}+\Delta B_{i}\right) u \\
\leq & s_{i}^{2}(x)-\delta_{i}^{2}(x)-x^{T} R_{i} x+2 x^{T} P_{i}\left(B_{i}+\Delta B_{i}\right) u .
\end{aligned}
$$

Noting that $\Delta B_{i}=B_{i} \Theta_{i}$ and $S_{i}=B_{i}^{T} P_{i}$, one can see that

$$
\begin{aligned}
\dot{L}_{i}(t) \leq & s_{i}^{2}(x)-\delta_{i}^{2}(x)-x^{T} R_{i} x+2 s_{i}(x) u_{i 1} \\
& +2 s_{i}(x) u_{i 2}+2 s_{i}(x) \Theta_{i}\left(u_{i 1}+u_{i 2}\right) .
\end{aligned}
$$

In the following, we first prove that

$$
s_{i}(x) u_{i 2}+\bar{d}_{i}\left|s_{i}(x)\right|\left|u_{i 1}+u_{i 2}\right| \leq 0 .
$$

Substituting (33) and (34) into the left side of above inequality, one has

$$
\begin{aligned}
s_{i}(x) u_{i 2}+\bar{d}_{i}\left|s_{i}(x)\right|\left|u_{i 1}+u_{i 2}\right| & \\
=- & \frac{1}{1-\bar{d}_{i}}\left(S_{i} B_{i}\right)^{-1}\left|s_{i}(x)\right| \rho_{i}+\bar{d}_{i}\left|s_{i}(x)\right| \\
\times & \mid\left(S_{i} B_{i}\right)^{-1} S_{i} A_{i} x+K_{i}\left(S_{i} B_{i}\right)^{-1} s_{i}(x) \\
& +\frac{1}{1-\bar{d}_{i}}\left(S_{i} B_{i}\right)^{-1} \operatorname{sign}\left(s_{i}(x)\right) \rho_{i} \mid .
\end{aligned}
$$

Noting that $S_{i} B_{i}=B_{i}^{T} P_{i} B_{i}>0$ and $\bar{d}_{i}<1$, we have

$$
\begin{aligned}
s_{i}(x) & u_{i 2}+\bar{d}_{i}\left|s_{i}(x)\right|\left|u_{i 1}+u_{i 2}\right| \\
\leq & -\left(S_{i} B_{i}\right)^{-1}\left|s_{i}(x)\right| \rho_{i}+\bar{d}_{i}\left(S_{i} B_{i}\right)^{-1}\left|s_{i}(x)\right| \\
& \times\left|S_{i} A_{i} x+K_{i} s_{i}(x)\right| .
\end{aligned}
$$

Since $\rho_{i}=\left|S_{i}\right|\left|D_{i}\right|\left|F_{i}\right||x|+\bar{d}_{i}\left|S_{i} A_{i}\right||x|+\bar{d}_{i} K_{i}\left|s_{i}(x)\right|$, it is easy to check that

$$
\rho_{i} \geq \bar{d}_{i}\left|S_{i} A_{i} x+K_{i} s_{i}(x)\right|
$$

It follows from (47) and (48) that (45) is established. Thus we have

$$
\dot{L}_{i}(t) \leq s_{i}^{2}(x)-\delta_{i}^{2}(x)-x^{T} R_{i} x+2 s_{i}(x) u_{i 1} .
$$

Substituting (33) into the above inequality (49) and noting that $K_{i}>\left(S_{i} B_{i} / 2\right), 2 K_{i} \alpha_{i}^{2} r_{i} Q_{i}+S_{i}^{T} S_{i} A_{i}+A_{i}^{T} S_{i}^{T} S_{i}>0$ and $s_{i}^{2}(x) \geq \alpha_{i} \delta_{i}^{2}(x)$, one can see that

$$
\begin{aligned}
\dot{L}_{i}(t) \leq & s_{i}^{2}(x)-\delta_{i}^{2}(x)-x^{T} R_{i} x+2 s_{i}(x) \\
& \times\left(-\left(S_{i} B_{i}\right)^{-1} S_{i} A_{i} x-K_{i}\left(S_{i} B_{i}\right)^{-1} s_{i}(x)\right) \\
= & -\left(S_{i} B_{i}\right)^{-1} x^{T}\left(2 K_{i} \alpha_{i}^{2} r_{i} Q_{i}+S_{i}^{T} S_{i} A_{i}+A_{i}^{T} S_{i}^{T} S_{i}\right) x \\
& -x^{T} R_{i} x \\
< & -x^{T} R_{i} x .
\end{aligned}
$$

After being forced into the inner sector of the $P_{i} R_{i}$-sliding sector and before moving out of the $P_{i} R_{i}$-sliding sector, the control input $u(t)$ is set equal to zero with $\sigma_{i}\left(s_{i}(x), \delta_{i}(x)\right)=0$. In this case, $\left|s_{i}(x)\right| \leq \delta_{i}(x)$ and

$$
\begin{aligned}
\dot{L}_{i}(t) & =x^{T}\left\{\left(A_{i}+\Delta A_{i}\right)^{T} P+P\left(A_{i}+\Delta A_{i}\right)+\sum_{j=1}^{\mathbb{N}} \lambda_{i j} P_{j}\right\} x \\
& \leq s_{i}^{2}(x)-\delta_{i}^{2}(x)-x^{T} R_{i} x \leq-x^{T} R_{i} x . \quad \forall x \in \mathcal{S}_{i} .
\end{aligned}
$$

Once the state trajectory of the plant runs out of the $P_{i} R_{i}$ sliding sector with zero control input, the control input (32)(34) will make it move back to the inside of the inner sector again, while the $P_{i}$-norm $|x|_{P_{i}}$ keeps decreasing.

Thus, from the above proof, one can see that it guarantees that the state trajectory of the plant moves from the outside of the $P_{i} R_{i}$-sliding sector into the inside of the inner sector and the Lyapunov function $L_{i}(x)=x^{T} P_{i} x$ keeps decreasing in the whole state space; then mean-square quadratic stability of the closed-loop system is established. 


\section{Illustrative Example}

Consider the Markov jump linear system with four operation modes:

$$
\begin{array}{cc}
A_{1}=\left[\begin{array}{ll}
1 & 0 \\
2 & 3
\end{array}\right], & A_{2}=\left[\begin{array}{cc}
0 & 1 \\
1 & -2
\end{array}\right], \\
A_{3}=\left[\begin{array}{cc}
2 & 0 \\
0 & -1
\end{array}\right], & A_{4}=\left[\begin{array}{cc}
3 & 1 \\
-1 & 2
\end{array}\right], \\
B_{1}=\left[\begin{array}{l}
1 \\
1
\end{array}\right], & B_{2}=\left[\begin{array}{l}
1 \\
1
\end{array}\right], \\
B_{3}=\left[\begin{array}{l}
2 \\
1
\end{array}\right], & B_{4}=\left[\begin{array}{l}
2 \\
3
\end{array}\right], \\
D_{1}=\left[\begin{array}{c}
0.1 \\
0
\end{array}\right], & D_{2}=\left[\begin{array}{l}
0.2 \\
0.5
\end{array}\right] \\
D_{3}=\left[\begin{array}{c}
0.2 \\
0.6
\end{array}\right], & D_{4}=\left[\begin{array}{l}
0.1 \\
0.5
\end{array}\right], \\
F_{1}=\left[\begin{array}{ll}
0 & -0.1
\end{array}\right], & F_{2}=\left[\begin{array}{ll}
1 & 0.3
\end{array}\right], \\
F_{3}=\left[\begin{array}{ll}
0.2 & 0.5
\end{array}\right], & F_{4}=\left[\begin{array}{ll}
0.1 & 0.1
\end{array}\right] .
\end{array}
$$

For simulation, we take $E_{1}(t, x)=0.1 \sin (t), E_{2}(t, x)=$ $0.1 \sin (2 t), E_{3}(t, x)=0.5 \sin (t), E_{4}(t, x)=0.4 \sin (2 t), \Theta_{1}=$ $0.2 \sin (2), \Theta_{2}=0.3 \cos (2 t), \Theta_{3}=0.2 \cos (t)$, and $\Theta_{4}=$ $0.2 \sin (2 t)$.

The transition rates matrix is

$$
\Lambda=\left[\begin{array}{cccc}
-1.5 & 0.2 & ? & ? \\
? & ? & 0.5 & 0.9 \\
1 & ? & -2 & ? \\
0.1 & \alpha & ? & \beta
\end{array}\right]
$$

where $0.5 \leq \alpha \leq 2$ and $-0.8 \leq \beta \leq-0.1$. Since there exist the completely unknown transition rates "?" and boundary known transition rates $\alpha$ and $\beta$, the reported methods in $[21,22]$ cannot work. Solving the LMIs conditions (21)-(22) in Theorem 11, one can obtain that positive definite matrices are as follows:

$$
\begin{gathered}
P_{1}=\left[\begin{array}{cc}
6.2534 & -6.4840 \\
-6.4840 & 18.4380
\end{array}\right], \quad P_{2}=\left[\begin{array}{cc}
6.8785 & -7.1343 \\
-7.1343 & 20.0458
\end{array}\right], \\
P_{3}=\left[\begin{array}{cc}
8.5811 & -9.6120 \\
-9.6120 & 15.6407
\end{array}\right], \quad P_{4}=\left[\begin{array}{cc}
6.1859 & -1.5374 \\
-1.5374 & 1.1026
\end{array}\right], \\
Q_{1}=\left[\begin{array}{cc}
6.9519 & -7.5088 \\
-7.5088 & 22.4304
\end{array}\right], \\
Q_{2}=\left[\begin{array}{cc}
13.4209 & -14.1627 \\
-14.1627 & 40.0263
\end{array}\right] \\
Q_{3}=\left[\begin{array}{cc}
11.4377 & -11.6823 \\
-11.6823 & 18.2712
\end{array}\right], \\
Q_{4}=\left[\begin{array}{cc}
10.3808 & -2.6205 \\
-2.6205 & 2.0926
\end{array}\right] .
\end{gathered}
$$

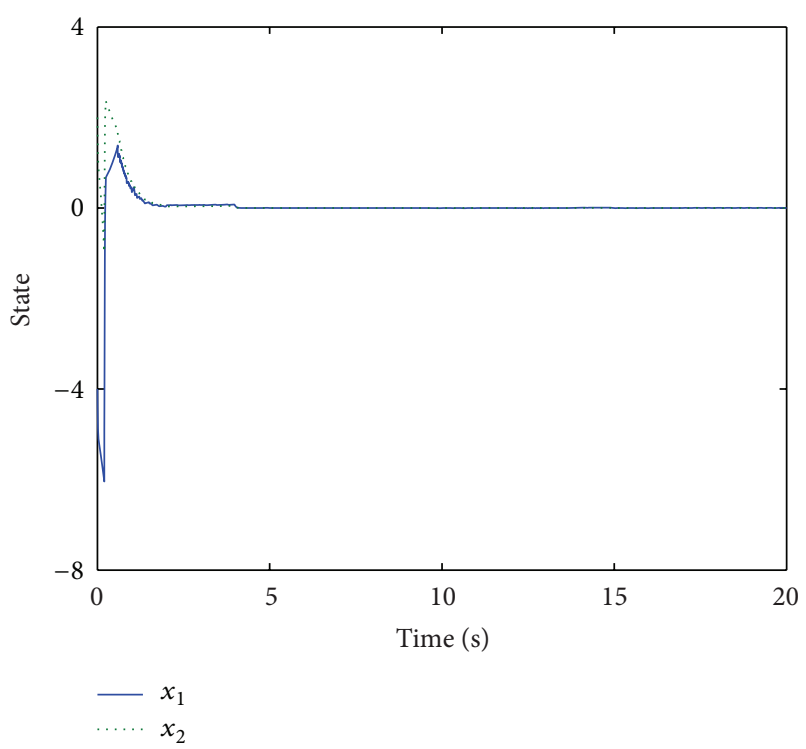

FIgURE 1: The response curves of the system states.

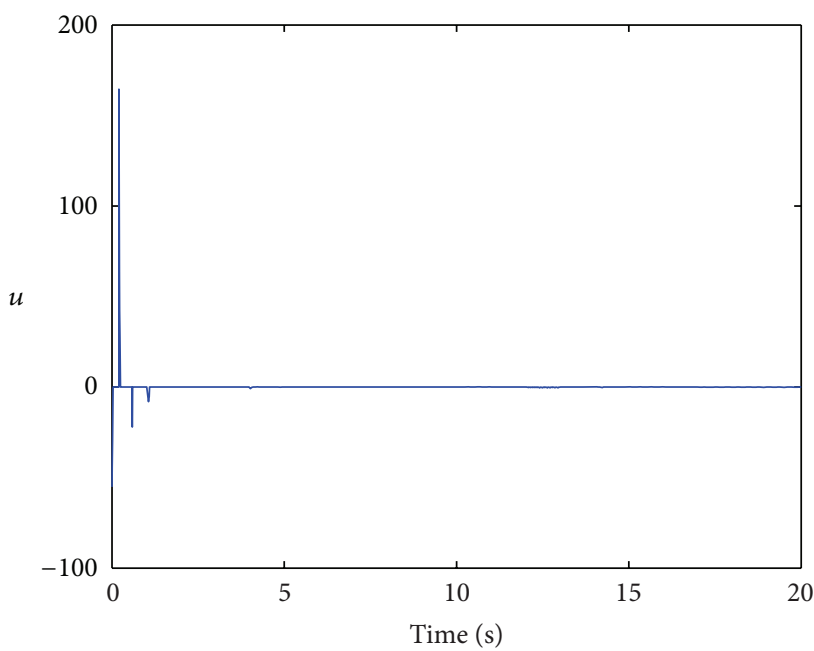

FIGURE 2: The response curve of the control input.

Selecting $\alpha_{1}=0.8, \alpha_{2}=0.9, \alpha_{3}=0.6, \alpha_{4}=0.6, r_{1}=0.8, r_{2}=$ $0.9, r_{3}=0.8, r_{4}=0.8, K_{1}=20, K_{2}=120, K_{3}=40, K_{4}=50$, then the condition in Theorem 12 is satisfied. For the purpose of simulation, let $\bar{d}_{1}=0.2, \bar{d}_{2}=0.3, \bar{d}_{3}=0.2$, and $\bar{d}_{4}=0.2$. Using these data, a simulation program has been done in Matlab. The simulation results are presented in Figures 1-3. It can be seen from Figure 1 that the system state can converge to the equilibrium point in less than 5 seconds. Figure 2 shows the response curve of the control input. One can easily see that no chattering phenomenon happens in the whole control process. The transition modes of system are given in Figure 3. It can be seen that the considered continuous-time Markov jump linear uncertain system is stochastic quadratic stable in spite of mismatched uncertainty and the transition rate matrix which covers completely known, boundary unknown, and completely unknown elements. 


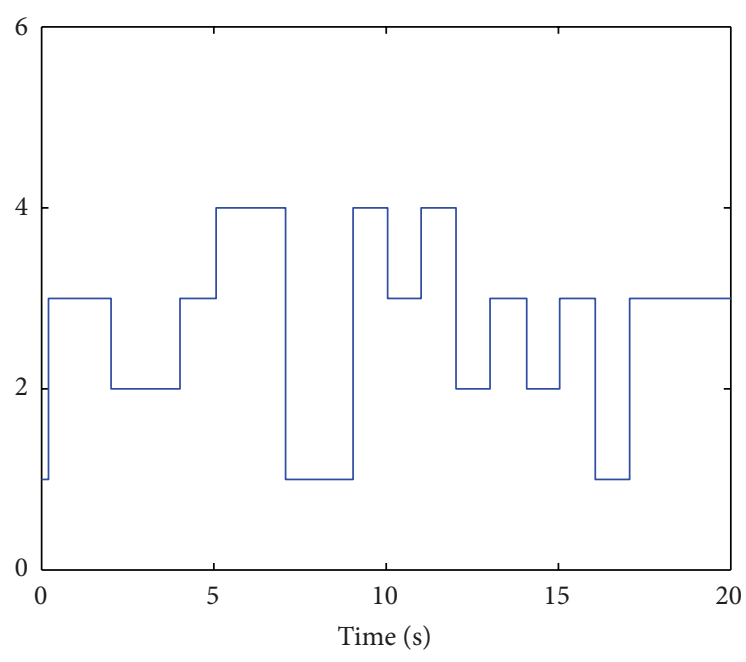

Figure 3: The response curve of the transition modes.

\section{Conclusions}

In this paper, the problem of designing sliding sector-based variable structure controllers is considered for Markov jump linear uncertain continuous-time time-invariant systems with unknown transition rates. First, the related notions about sliding sector for continuous-time MJLSs are presented and sufficient conditions for the design of the sliding sector are given by LMIs. Second, the variable structure control law is presented to ensure the mean-square quadratic stability of the closed-loop system, and simulation results finally have verified the effectiveness of the proposed method. In our future research, how to design dynamic output feedback variable structure controller based on sliding sector technique and considering the applying of the sliding sector technique in industrial robotic systems are interesting work. Finally, the authors would like to thank the associate editor and all the reviewers for their constructive comments and suggestions for improving this paper.

\section{Acknowledgments}

This work was supported in part by the Funds of National Science of China (nos. 11301276, 61104123), the Natural Science Funds of Jiangsu Province (BK20130984, BK20131000), the Scientific Research Foundation of Nanjing University of Information Science \& Technology (S8112075001, S8112081001), and Laboratory Open Fund of Nanjing University of Information Science \& Technology (N1081005112).

\section{References}

[1] L. Dai, D. Huang, and Z. Li, "Sliding mode control of hybrid joints for wheeled mobile manipulators with Markovian switching and bounded torques," Intelligent Robotics and Applications, vol. 6424 , no. 1, pp. 171-182, 2010.

[2] L. Ding, H. Gao, K. Xia, Z. Liu, J. Tao, and Y. Liu, "Adaptive sliding mode control of mobile manipulators with markovian switching joints," Journal of Applied Mathematics, vol. 2012, Article ID 414315, 24 pages, 2012.
[3] E. K. Boukas, Stochastic Switching Systems: Analysis and Design, Birkhauser, Berlin, Germany, 2005.

[4] E. K. Boukas and H. Yang, "Stability of discrete-time linear systems with Markovian jumping parameters," Mathematics of Control, Signals, and Systems, vol. 8, no. 4, pp. 390-402, 1995.

[5] J. X. Dong and G.-H. Yang, "Robust $H_{2}$ control of continuoustime Markov jump linear systems," Automatica, vol. 44, no. 5, pp. 1431-1436, 2008.

[6] M. G. Todorov and M. D. Fragoso, "On the stability radii of continuous-time infinite Markov jump linear systems," Mathematics of Control, Signals, and Systems, vol. 22, no. 1, pp. 23-38, 2010.

[7] O. L. V. Costa and E. K. Boukas, "Necessary and sufficient condition for robust stability and stabilizability of continuous-time linear systems with Markovian jumps," Journal of Optimization Theory and Applications, vol. 99, no. 2, pp. 359-379, 1998.

[8] Z. Wang, J. Lam, and X. Liu, "Exponential filtering for uncertain Markovian jump time-delay systems with nonlinear disturbances," IEEE Transactions on Circuits and Systems II, vol. 51, no. 5, pp. 262-268, 2004.

[9] S. Xu, T. Chen, and J. Lam, "Robust $H_{\infty}$ filtering for uncertain Markovian jump systems with mode-dependent time delays," IEEE Transactions on Automatic Control, vol. 48, no. 5, pp. 900907, 2003.

[10] M. Karan, P. Shi, and C. Y. Kaya, "Transition probability bounds for the stochastic stability robustness of continuousand discrete-time Markovian jump linear systems," Automatica, vol. 42, no. 12, pp. 2159-2168, 2006.

[11] J. L. Xiong, J. Lam, H. J. Gao, and W. C. Daniel, “On robust stabilization of Markovian jump systems with uncertain switching probabilities," Automatica, vol. 41, no. 5, pp. 897-903, 2005.

[12] L. Zhang, E.-K. Boukas, and J. Lam, "Analysis and synthesis of Markov jump linear systems with time-varying delays and partially known transition probabilities," IEEE Transactions on Automatic Control, vol. 53, no. 10, pp. 2458-2464, 2008.

[13] L. Zhang and E.-K. Boukas, "Stability and stabilization of Markovian jump linear systems with partly unknown transition probabilities," Automatica, vol. 45, no. 2, pp. 463-468, 2009.

[14] L. Zhang and J. Lam, "Necessary and sufficient conditions for analysis and synthesis of markov jump linear systems with incomplete transition descriptions," IEEE Transactions on Automatic Control, vol. 55, no. 7, pp. 1695-1701, 2010.

[15] P. Y. Richard, H. Cormerais, and J. Buisson, "A generic design methodology for sliding mode control of switched systems," Nonlinear Analysis: Theory, Methods and Applications, vol. 65, no. 9, pp. 1751-1772, 2006.

[16] G. S. Tombul, S. P. Banks, and N. Akturk, "Sliding mode control for a class of non-affine nonlinear systems," Nonlinear Analysis: Theory, Methods and Applications, vol. 71, pp. 1589-1597, 2009.

[17] M. Roopaei and M. Z. Jahromi, "Chattering-free fuzzy sliding mode control in MIMO uncertain systems," Nonlinear Analysis: Theory, Methods and Applications, vol. 71, no. 10, pp. 4430-4437, 2009.

[18] Y. Niu, D. W. C. Ho, and J. Lam, "Robust integral sliding mode control for uncertain stochastic systems with time-varying delay," Automatica, vol. 41, no. 5, pp. 873-880, 2005.

[19] Y. Niu, D. W. C. Ho, and X. Wang, "Sliding mode control for Itô stochastic systems with Markovian switching," Automatica, vol. 43, no. 10, pp. 1784-1790, 2007.

[20] B. C. Zheng and G. H. Yang, "Sliding mode control for Markov jump linear uncertain systems with partly unknown transition rates," International Journal of Systems Science, 2013. 
[21] P. Shi, Y. Xia, G. P. Liu, and D. Rees, "On designing of slidingmode control for stochastic jump systems," IEEE Transactions on Automatic Control, vol. 51, no. 1, pp. 97-103, 2006.

[22] S. Ma and E.-K. Boukas, "A singular system approach to robust sliding mode control for uncertain Markov jump systems," Automatica, vol. 45, no. 11, pp. 2707-2713, 2009.

[23] C. Edwards and S. K. Spurgeon, Sliding Mode Control: Theory and Applications, Taylor and Francis, London, UK, 1998.

[24] J. Y. Hung, W. B. Gao, and J. C. Hung, "Variable structure control: a survey," IEEE Transactions on Industrial Electronics, vol. 40, no. 1, pp. 2-22, 1993.

[25] V. I. Utkin, Sliding Modes in Control and Optimization, Springer, Berlin, Germany, 1992.

[26] K. Furuta and Y. Pan, "Variable structure control with sliding sector," Automatica, vol. 36, no. 2, pp. 211-228, 2000.

[27] I. R. Petersen, "A stabilization algorithm for a class of uncertain linear systems," Systems and Control Letters, vol. 8, no. 4, pp. 351357, 1987.

[28] R. A. Horn and C. R. Johnson, Matrix Analysis, Cambridge University Press, 1985. 


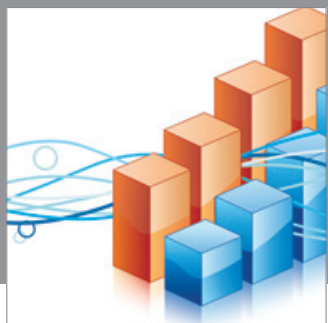

Advances in

Operations Research

mansans

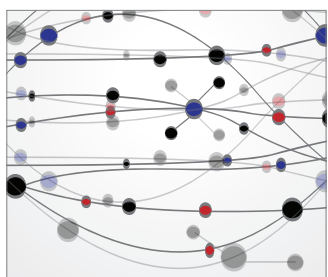

The Scientific World Journal
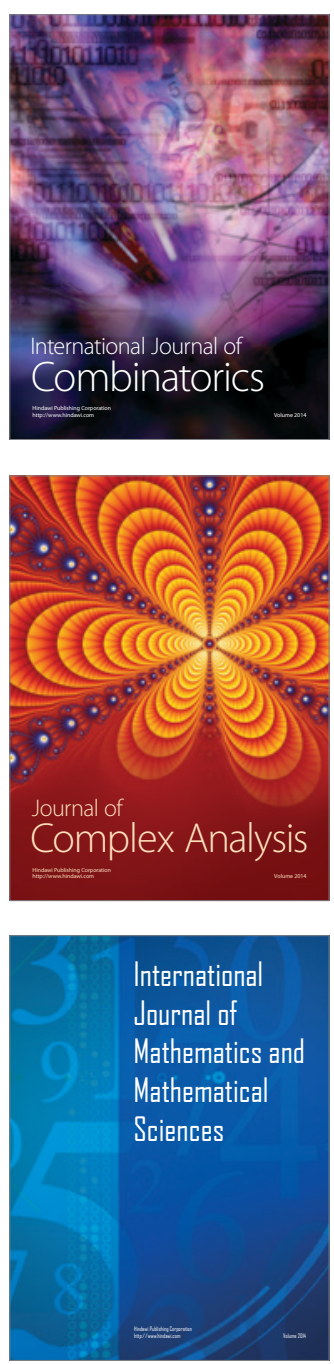
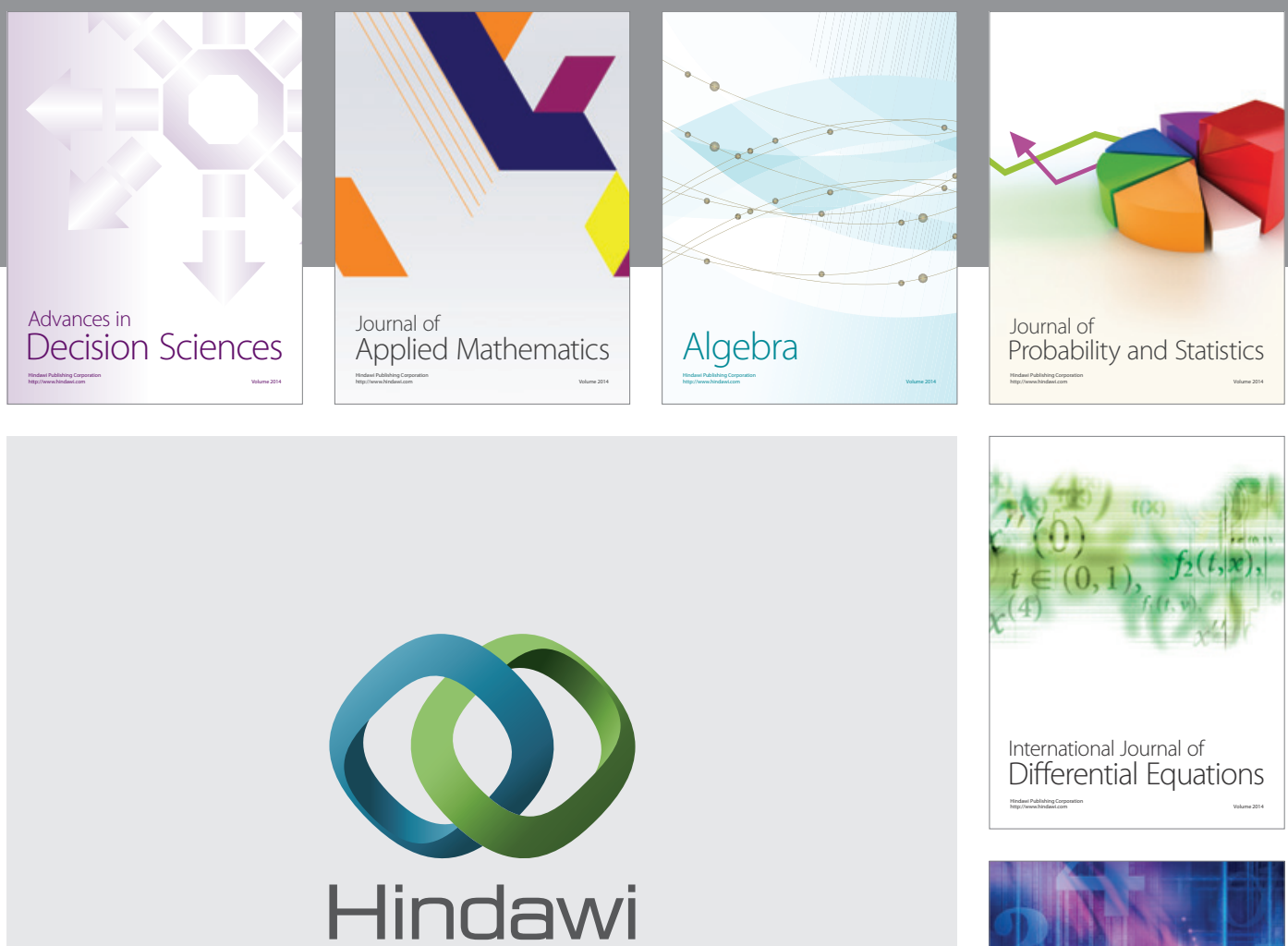

Submit your manuscripts at http://www.hindawi.com
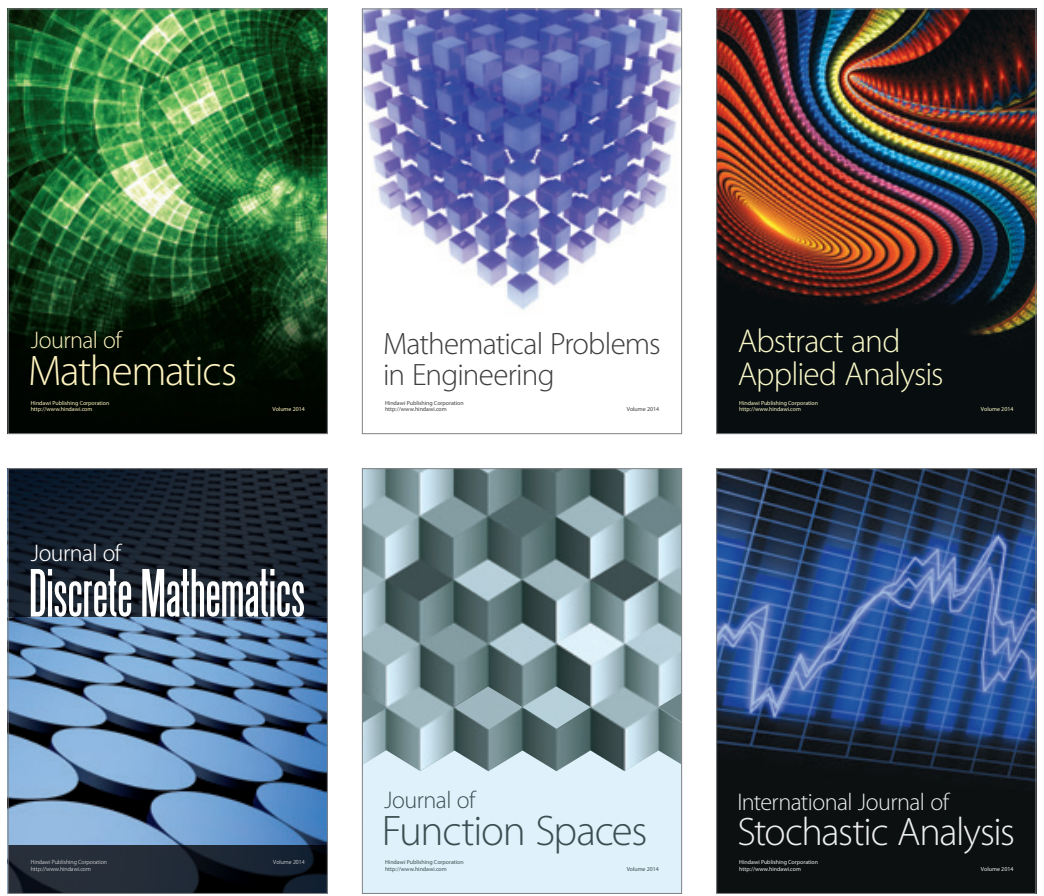

Journal of

Function Spaces

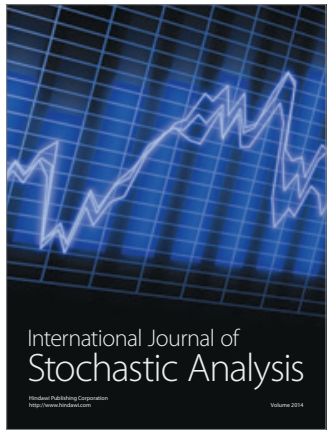

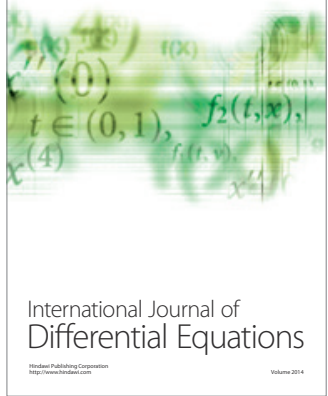
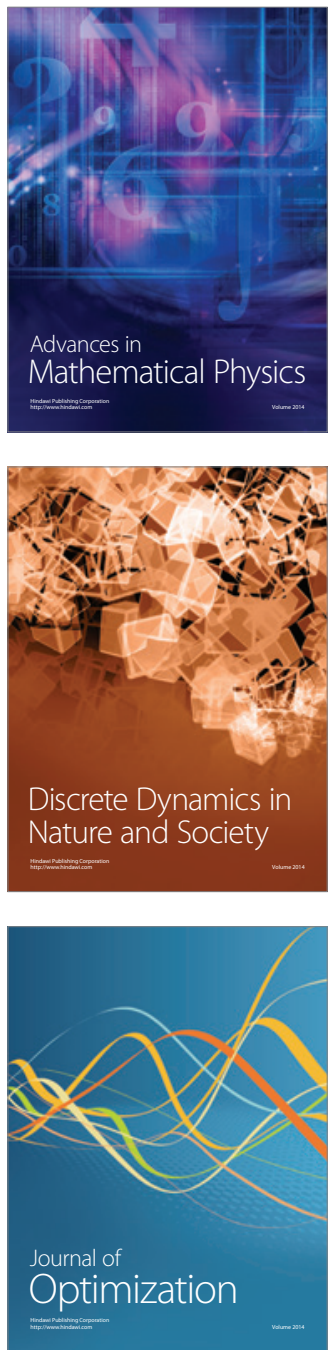